\title{
MARIANA MAGGIO (2018), REINVENTAR LA CLASE EN LA UNIVERSIDAD, PAIDÓS, BUENOS AIRES, 183 PÁGINAS
}

\author{
Alexis Chausovsky* \\ Facultad de Ciencias de la Educación de la Universidad Nacional de Entre Ríos, Argentina \\ alexischausovsky@gmail.com
}

Recibido: 16/08/2019 Aceptado: 26/20/2019

Reinventar la clase en la universidad nos propone resquebrajar los cimientos de la didáctica clásica y a generar nuevas iniciativas en un escenario que ofrece desafíos incesantes y heterogéneos. La propuesta de Mariana Maggio se fundamenta en dos nociones clave: enseñanza poderosa e inclusión genuina. La primera procura interrogar la enseñanza para su actualización, desarrollando interrogantes abiertos para una práctica que conmueva; la segunda implica reconocer cómo se construyen los conocimientos de los docentes, advertir las transformaciones de la sociedad y la enseñanza en el escenario contemporáneo.

El primer capítulo toma distancia de las modalidades de la clase expositiva caracterizada por una explicación unilateral de docentes que con ella pretenden reparar la selección bibliográfica fragmentaria de pequeños textos inconexos que conforma los programas de estudios. Maggio busca desestabilizar el dominio de aquello que Edith Litwin bien denominó "secuencia lineal progresiva" de las clases. Desde la mirada de la enseñanza poderosa, las clases memorables se basarían en el abordaje teórico actual de las problemáticas correspondientes, mirando en tiempo presente para buscar una lectura original. La clase sería un espacio de invención, partiendo de las investigaciones que en ella misma se lleven a cabo. La autora llega a afirmar que "cada clase es un invento" y pone en un tembladeral las rutinarias clases idénticas en diferentes ciclos lectivos, indiferentes a lo debatido en los medios de comunicación y las redes sociales o las situaciones institucionales.

El segundo capítulo gira alrededor de la noción de inmersión, signada por la experiencia de constante participación en los nuevos formatos narrativos. La inmersión, rasgo cultural de una época con alta disponibilidad tecnológica, combina la televisión, los juegos en línea y las plataformas como Netflix y modifica los modos de relacionarse con las historias. Maggio valora el modelo de las nuevas series para demostrar la potencia de las preguntas que enhebran los diferentes episodios y mantienen la atención del público. A su vez pone de relieve el planteo de problemas que no pueden ser estudiados por una sola teoría, la riqueza del contexto en que se incluyen, el cuestionamiento al tiempo lineal en pos de saltos e interrupciones, los dilemas que llevan al extrañamiento, la anticipación temática y la hibridación de géneros y tramas. Así, propone romper las concepciones estrechas que delimitan la educación al tiempo y al espacio del aula.

El tercer capítulo demuestra que las condiciones para una clase distinta se han de sostener en la transformación del programa de estudios, que podría basarse principalmente en preguntas y no sólo en objetivos a cumplir. Ello permitiría el abordaje de temas relevantes en la agenda actual como los videojuegos y la gamificación-. Incluso en un mundo con amplia disponibilidad tecnológica, la autora también destaca la lectura de libros completos y en profundidad -observando de hecho la posibilidad de convocar a sus autores a las clases- para que se amplíen las condiciones para un conocimiento novedoso, sin separar al libro de la praxis, sino enalteciendo el poder de alterar los tiempos y los espacios de la clase.

\footnotetext{
* Master en philosophie et critiques contemporaines de la culture (Université Paris VIII) y Magíster en Comunicación y creación cultural (Fundación Walter Benjamin, Universidad CAECE, Buenos Aires), Licenciado y Profesor en Comunicación Social (Universidad Nacional de Entre Ríos). Profesor de la Licenciatura y el Profesorado en Comunicación Social de la Facultad de Ciencias de la Educación de la Universidad Nacional de Entre Ríos y de la Facultad de Ciencias de la Gestión de la Universidad Autónoma de Entre Ríos. Integrante de la Editorial de la Universidad Nacional de Entre Ríos EDUNER.
} 
Esta tentativa requiere conformar otros modos de evaluación. Como dice Maggio en el cuarto capítulo, se trata de tener presente el sentido moral del acto de evaluar, que puede ser entendido incluso como una creación. Para fundamentarlo, expone las evaluaciones de la cátedra a su cargo, Tecnología Educativa de la Licenciatura en Ciencias de la Educación (UBA), entre 2011 y 2017, que involucran el estudio de casos, la reflexión de situaciones cotidianas, el uso de las redes sociales como herramientas mediadoras para construir conocimiento colaborativamente, la combinación de la resolución de problemas de parte de los estudiantes que se ponen en el lugar de tecnólogos educativos. Se entiende a la evaluación a partir de la coherencia con la propuesta didáctica, en pos inserciones críticas en las prácticas, que implique negociaciones colectivas y busque propiciar la inclusión de los estudiantes.

La propuesta didáctica con la que será coherente la evaluación es el centro del quinto capítulo. Estamos hablando, siguiendo a Maggio, de una "didáctica en vivo", que procura transformar el conocimiento teórico mientras se recrea la práctica de enseñanza. La didáctica en vivo es aquella que sostiene prácticas que dan cuenta de las transformaciones culturales recientes y que aún no está construida. La clase universitaria mirada desde una didáctica en vivo se expresa, por ejemplo, divisando las tendencias actuales, reconstruyendo las clases en redes sociales para la producción teórica colectiva. Es una propuesta que va más allá del aula, de una cátedra o una carrera específica y que sugiere, como dice la autora en el sexto capítulo, que las clases se vuelvan experiencias que vale la pena vivir. Dados los peligros que aquejan a la educación pública, que van desde las posibilidades restringidas para su ingreso hasta los riesgos de su deserción, es necesario que las clases sean extraordinarias, únicas. Ello implica combinar los formatos escritos y multimedia hacia producciones creativas conjuntas entre docentes y estudiantes, advirtiendo a su vez la vigencia y los alcances del pensamiento de autores y autoras en cada área de conocimiento.

El séptimo capítulo establece que lo propuesto en el libro sólo tiene como condición que la enseñanza sea entendida como proyecto colectivo. Gracias a la trama de atravesamientos de las tecnologías de la información y la comunicación y la emergencia de la idea de inteligencia colectiva, se abren nueva formas de cultura participativa que a su vez reconfiguran el trabajo de los equipos de cátedra y la participación de los estudiantes. En un mundo de "humanidad aumentada", interconectada, hibridada con sistemas que guían las conductas individuales y colectivos, hacer un uso conjunto horizontal, con diversos aportes, para |debatir, criticar e imaginar.

Con este recorrido, el libro llama a la lectura, a la reflexión y a la renovación de las prácticas que se han legitimado por su propia reiteración y que se toman como naturales o ineludibles. Nos encontramos ante una producción indispensable, tanto para las discusiones teóricas en didáctica como para reconfigurar las clases y los modos de concebir la educación universitaria en su conjunto. 